\title{
Iris Recognition based Biometric identification using Neural Networks
}

\author{
Reend Tawfik Mohammed, Shafqat Ul Ahsaan, Harleen Kaur* \\ \{harleen.unu@gmail.com\} \\ Department of Computer Sciences and Engineering, School of Engineering Sciences and Technology \\ Jamia Hamdard, New Delhi, India
}

\begin{abstract}
Iris identification is one of the striking biometric identification procedures for recognizing human beings based on physical behavior. The texture of the iris is unique and its' anatomy varies from individual to individual. The Irisrecognitionsystem works in three stages: In the first phase, the iris is localized and stored in the database. In this paper, we have elaborated on multiple methods for the detection of iris-based neural networks. The Iris is extracted from an image database and in the second pass normalization is performed and next is the enhancement. There are many biometric techniques available for distinguishing between the physical or behavioral characteristics of human beings. The physical features of human beings are unique, and they never change this has conducted a significant development in the field of iris recognition. Iris detection is considered to be a very reliable field because of its texture's random variation. This paper highlighted the use of Neural Networks for the recognition of the iris. We focus on the current research works that have been carried in the field of bioinformatics used for the identity of an individual. It includes many methods like localization, normalization, comparison, and encoding. This recognition field has many practical and research applications. The region of the Iris is localized in the dataset for the Iris image is generated, and later pattern recognition is carried out.
\end{abstract}

Keywords: Biometrics, Iris Recognition, Normalization, Localization, Neural Networks, Iris Encoding, Neural Network, Back Propagation Neural Network

\section{Introduction}

Biometrics tools play a key role in the field of information security by using specific anatomical features for the human body such as fingerprints, iris, retina, hand morphology, etc. [1]. Iris recognition is considered as one of the most potent biometric identification ever, as it's a dynamic subject matter in research and practical appliance among the broad sets of biometrics options[2]. Iris is the expandable, pigmented connective tissue that controls the pupil. Iris constitutes the main part of the eye and it remains unchanged till death. There is no effect of the environmental changes on the texture and structure of the iris [2]. Because of this uniqueness of their stability and reliability, we consider it the most important biometrics. The procedure 
used for the Iris identification mainly includes the capturing, pre-processing, and acceptance of the Iris in the digital image. Pre-processing includes Iris localization, normalization, and enhancement; every step uses a different algorithm. There are quite a lot of techniques that have been framed for the localization of an Iris such as gradient basal Hough transform for the localization of Iris[3], circular Hough transform is useful to uncover the Iris circles and complete the localization. The canny operator is used to trace the pupil margin, but these methods require enough time to determine the iris. Multiple biometric processes can be used to detect and verify the identity of an individual; the important ones are described below:

i. Fingerprint verification: A person's fingerprint is taken, and it is compared with already existing prints in the database. e.g., Banks.

ii. Hand recognition: It reads a person's hand or finger for access. It uses it for authentication for granting access.

iii. Voice verification: It uses a small voice sample like a word, key, or even a number usually used in A.T.M.s and biometric A.T.M.s and uses customers' voice for processing.

iv. Iris scan: It uses the human eye and analyses and scans the Iris, which exists in the colored tissue surrounding the pupil of an eye. It is a traditional camera element and requires no physical.

v. Facial recognition: It analysis the character of the person's face. If there is a match, access is granted. A person faces a digital camera at a near distance so that the overall face structure like eyes, nose, mouth, jaw can be easily measured.

vi. Signature recognition: The speed, slant direction of writing is analyzed and used for identification.

vii. Vascular pattern: It uses wings present in hands or faces the thickness and texture of wheels used for analysis.

\section{Related Works}

Tobji, R et al. [4] introduced an iris recognition algorithm through Fully Convolutional Network (FCN) and Multi-Scale Convolutional Neural Network (MCNN). The authors' introduced an approach that uses feature extraction and classification for iris detection. The algorithm provides the best results for a rating in contrast with other state-of-the-art iris recognition methods. Zhang, W, and Jia, H [5] introduced an iris recognition method using Convolutional Neural Networks $(\mathrm{CNN})$. Pre-processors are being used in this framework for the extraction of the Iris, feature extraction is used and the Iris is divided into eight rectangular sub-regions and fed to the developed model. The recognition rate for these methods ranges up to $99 \%$. Sequeira et al. [6] introduced a novel cross-spectral and cross-eyed dataset for iris and put the efforts on the crossspectral iris challenge problem having an error rate of less than $4 \%$. However, this work is novel and is primarily not compared with other algorithms. Mozumder, A.I., and Begum, S. A., [7] presented a method that is comprised of three stages: the first part is segmentation and normalization of Iris. It is done by using Quantum-behaved Particle Swarm Optimization (QPSO), Discrete Cosine Transform (DCT) is used for feature extraction and Fuzzy inference scheme (FIS)and Modular Neural Network (MNN) based on Score Level Fusion (SLF) for the 
classification of iris. The proposed method attains $98 \%$ accuracy for the identification and verification of Iris, respectively.

Hajari, K. et al. [8] introduced two novel algorithms: the first one works on noise removal from iris images, and the next one for feature extraction using a composite method like Gray Level Co-occurrence Matrix (GLCM) and Local Binary Pattern (LBP). The authors' performed the experimental results using CASIA and MMU datasets for iris identification. The structure can execute in a raucous imaging environment and achieved the iris identification of (96\%). Kulkarni, S. B. et al. [9] developed a method that uses Circular Hough transform (CHT) for picture segmentation and, after that, divided the iris image into rectangular blocks of fixed size via Daugman's Rubber sheet model (DRSM). The feature extraction for Iris is performed by deploying the CMYK color model, and a feature vector $(\mathrm{FV})$ is created using a $2 \mathrm{D}$ Walsh Hadamard transform (2DWHT). Finally, the classification is performed employing Artificial Neural Networks (ANN). The results are accomplished by using the UBIRIS database, and the framework attained the classification accuracy rate of $87 \%$.

Chowhan, S.S. et al.[10] developed an iris detection and classification system that includes primarily three stages: normalization, feature extraction (FE), and classification. In their work, they deployed fuzzy min-max (FMN) neural network classification using Patrick Simpson's hyper box fuzzy sets. The authors' have used the CASIA. the dataset containing 756 images and compared the performance of the proposed system based on similarity measures for different classifiers. Bazrafkan, S. et al.[11] utilized a feed-forward neural network with backpropagation learning and learning vector quantization network (LVQ) for the detection of iris. The introduced system works in three phases: the first stage is segmentation and the next part is $2 \mathrm{D}$ distinct wavelet transform (DWT) for mining features utilizing separate wavelet secondary bands and the third and last phase is classification. In their work, they have deployed the proposed system on the UBIRIS database and the Bath database. The results obtained showed that the accuracy of image recognition is $90 \%$, and it depends on the quality of images.

Hilal, A. et al. [12] offered a novel technique for the normalization of the iris. In their work, they have used Daugman's normalization technique for parabolic function, Gabor filters for feature extraction, and artificial neural network for classification problems. To evaluate the performance of the system, the CASIAV3 database that contains iris images is used. The parabolic normalization approach enhances the results to a more excellent value in terms of precision for error rate (ER) operating point and receiver operating trait curves. Peter, A. et al [13] pioneered an efficient iris detection method consisting of four phases. In the first phase, the Circular Hough Transform (CHT) is used for iris localization, followed by normalization of iris into constant measurements. In the third phase, the Gray-Level Co-occurrence Matrix (GLCM) is used for examining iris textures and Circular Hough Transform (CHT) for feature extraction. In the last phase, Artificial Neural Network (ANN) with Back Propagation (BP) is used for the classification of iris. The proposed system produced the best results in terms of accuracy and attained an accuracy of $97 \%$.

\section{Iris recognition system}

The Iris identification system in neural networks consists of Iris image acquisition, which means capturing the image of the eye, lighting system, positioning system, and the real capture system $[14,15]$. The Iris identification involves pre-processing and neural networks during the 
Iris acquisition; the Iris image must be clear and sharp in the input sequence[16]. The addition of images considers the first step of the recognition system. This step isn't easy, but it is complicated because of the differences in the size of people's eyes and different colors of iris from one person to another. The average distance to capture should be 2-3 feet, and the average time is 1-2 seconds. The same person's result in image acquisition could be different due to ambient conditions such as light influencing specific distance separation and positioning [17]. Pre-processing is a very critical step in the Iris recognition system to get rid of the image noise and prepare the Iris image for the next phase, such as eye image extraction and normalization functionality. Normalization is to convert the image into a form that needs to be enhanced. After the enhancement, the matrix that describes the Iris image's grayscale values is represented. This matrix becomes the set for neural network training data. The Iris detection system contains two operational modes; training mode and online mode. Training of a recognition system is done at the first stage using grayscale image meaning. After the training phase in the online neural network, the neural network is equipped with Iris's picture and distinguishes the patterns that belong to the Iris of a person [16,17]. According to JohnDaugman's algorithm, the model of an eye image can be extracted from the image of an eye and encoded that code will be matched. Comparison results can reflect the amount of difference between the systems being compared. Therefore it can be inferred if the patterns of the reference eyes belong to the same or different person or not.

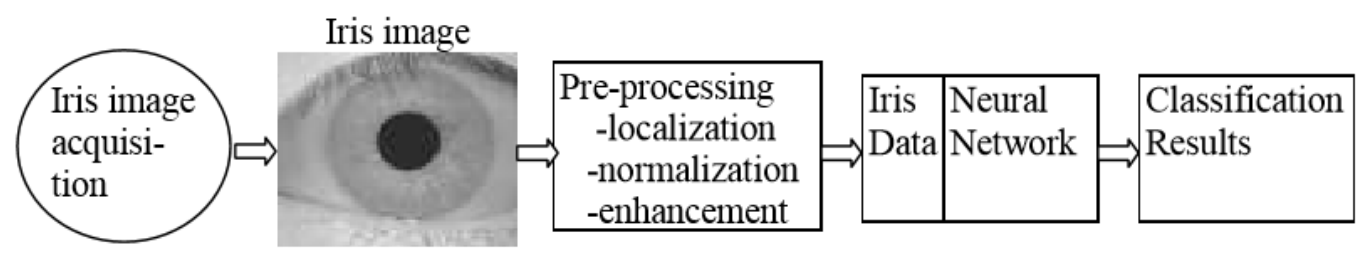

Figure 1. The architecture of the iris recognition system

\subsection{Iris localization}

The anatomy of the eye includes not only the Iris but the pupil, eyelids, sclera are the main elements of the eye. The segmentation needs to be completed to localize and take out the Iris from the image data. The main thing in localization is to restrict the Iris from the other un-useful parts. Many frameworks have been designed for the Iris localization, and the most commonly used technique is circular Hough transform, which plays a key role in detecting the radius and center of the iris image[14,18]. The picture of the iris is first transformed into a binary form by adding an acceptable threshold value. This is followed by an edge detector algorithm such as a canny edge[19]. Eventually, the Hough Transform is applied to the edge picture to discover a 
pupil circle. It results in faster and more accurate identification of pupils. Edge Detection: Some of the techniques used to identify edges and canny edge detection methods, but as this way is time-consuming to locate the boundaries, so we are seeking to find a faster algorithm to detect the Iris. John Daugmanpresented a way to exclude eyelashes by using statistical inference next to camera noise and eyelid occlusion. The eyelash occlusion usually has random and intricate shapes that combine to form a mass of intersecting elements rather than pure hair as a standard that could be detected by elementary shape models [20].

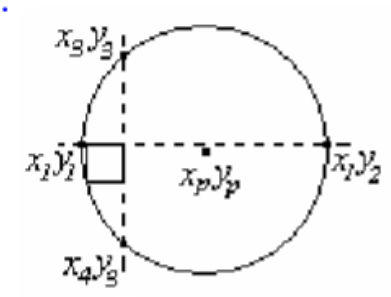

Finding the centre of the pupil

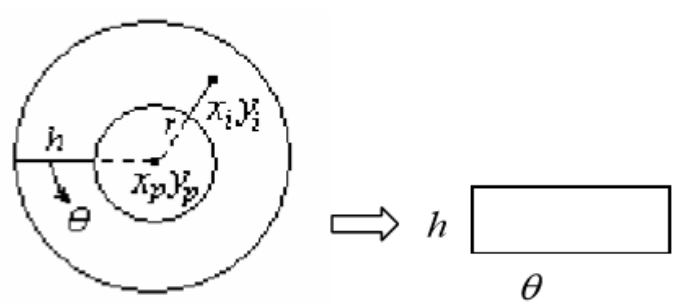

Normalization of the iris

\subsection{Iris normalization}

Different people have different Iris sizes. Sometimes there are changes in the eye because of the distance from the camera, illumination variation, or another factor. The factors above can affect the accuracy of this technique to avoid all these issues and make a better prediction. Normalization of Iris needs to be done [16]. The standardization of the Iris is used to change the texture of the Iris from Cartesian to polar coordinates. The procedure for the normalization will generate iris regions with the same unvarying proportions so that the copy of the same iris in varying conditions will possess similar features at the same time, and this process is done by using the following operation

$$
\begin{array}{r}
\emptyset \in[0,2 \pi] r \in[R p, R L(\theta)] \\
x i=x p+r \cdot \cos (\theta) ; y i=y p+r \cdot \sin (\theta)
\end{array}
$$




\subsection{Image enhancement}

To eliminate high-frequency noise, and to make advancement in the contrast of the iris image that needs to be detected. Mostly the images which are obtained are larger and unsuitable to be fed in the neural network, so it needs to lessen its dimensions. This reduced dimension prototype thus obtained will serve as a function vector for the artificial neural network [21].

\section{Neural Network(NN) and Artificial Intelligence(A.I)}

Unique mechanisms that are the basis of human knowledge have been identified from the scientific and philosophical studies conducted over the centuries. Inspired by their processes, computers could be produced to mimic part of those mechanisms [22]. The problem is that they have still not managed to emulate and incorporate all of them, so Artificial Intelligence. Similar to the structure of biological neurons, ANNs describe the neuron as a central processing unit that performs a mathematical operation to produce one output from a group of inputs. A neuron's production is a function of the weighted input sum plus the bias. The neural network in iris recognition works by changing the weight value during the training stage to lessen the errors among the real and the desired performance pattern of an eye [23].

\subsection{Back Propagation Neural Network (BPNN) based iris pattern recognition}

Back Propagation Neural Network (BPNN) is a methodical approach for multilayer training of artificial neural networks [20]. Back-propagation is considered as an efficient method for changing the weights of a feed-forward network, with differentiable activation function units, to learn a training set of input-output. It gives a decent way to minimize the total squared error of the network-computed output. The Back Propagation Neural Network is trained by supervised learning methods. The composition of the BPNN consists of an input layer, a hidden layer, and the output layer [22]. The BPNN used for iris identification takes the image of the iris as input via the input layer for the characterization of the iris image. For the processing, the number of nodes in the hidden layer is the set of trails and errors during the training phase. The output layer constitutes the number of subjects that are present in the data set [23].

The algorithm for using BPNN for iris identification is given below:

1 Load Iris data set for different subjects containing (contains vector function values from 0 to 1 for different subjects).

2 Perform the training and testing randomly of the data set after normalization.

3 Build an NNstructural design of input, hidden, and output layer. The number of nodes possessed by the input layer depends on the value of feature vectors that the dimension of the characteristic vector that distinguishes the information about the iris image, followed by the hidden layer. There is a single node at the output layer. Initialize all contact weights at random within a given range.

4 Train the network on the training set using the Back Propagation algorithm until the error for a certain number of user-specified training times to be neglected. 
5 Upload the test data to the trained network and determine the results.

\subsection{Feedforward neural networks}

Within neural networks, the signal flow can be either within one direction or in recurrence. In the first example, we call the feed-forward neural network architecture, since the input signals are fed into the input layer. Then they are forwarded to the next layer after processing, as shown in the figure below. Once the output is reached, we calculate the error (predicted output minus initial production) at the last layer (the output layer). The weights and biases used in forwarding propagation need to be corrected with this error where they use the derivative function. The weight to adjust is determined by gradient descent $[22,24]$.

\section{Methodology}

Iris recognition system Methodology uses canny edge detection with Hough transform to segment iris images to find the Iris and eliminate the noise. The normalization method is used to un-wrap the iris to obtain polar coordinates when the image is shown. Iris recognition system works by localizing the Iris from an eye image and apply circular Hough transform and canny edge detection then normalizing the eye to change the texture of the Iris from Cartesian to polar coordinates. The last step to do the image-enhancement to eliminate the noise from the image before we feed it to the neural network.BPNNalgorithms are to be used for training the iris image after localization, normalization, and enhancement. Thus feature encoding is carried out by breaking the two-dimensional normalized Iris pattern into one-dimensional wavelets, and then these signals are convoluted into one-dimensional Gabor wavelets. To order to find the best performance, the single value decomposition is applied to these coefficients for comparison [25]. The following formula is used to do the feature extraction in the iris image. Iris recognition system can gain speed, equipment simplicity, precision, and learning skills with the growth of an Artificial Intelligence algorithm. Artificial neural networks (ANN) or connection systems are programming systems that are vaguely inspired by the physical neural networks that make up animal brains. An ANN is based on a series of linked units or nodes called artificial neurons that loosely replicate a physical brain's neurons. As with the synapses in a biological brain, each connection will send a signal to other neurons. An artificial neuron that receives a signal then processes it and can signal associated neurons. Artificial neural networks (ANNs) are simple, core nervous system-based models. ANN models have compelling techniques for building powerful smart computers.ANN systems have been developed in past years for deep learning, image processing, speech recognition, data encoding, and prediction

$$
G(X, Y ; \theta, \omega)=e-\frac{1}{2\left[\frac{x^{\prime 2}}{\delta^{\prime 2}}+\frac{y^{\prime 2}}{\delta^{\prime 2}}\right]} e^{(i \omega(x+y))}
$$


where

$$
x^{\prime}=x \cos \theta+y \sin \theta
$$

$$
y^{\prime}=y \cos \theta+x \sin \theta
$$

After the application of equation (2), Gabor Filter is to be used to get the optimum resolution in both the domains [26]. As the information about time is missing and it's challenging to get a specific frequency that occurs in Fourier Transform. So Gabor Filter fits very well for the resolution analysis in both domains [27].

\section{Conclusion}

In this paper, we have elaborated on multiple methods for the detection of iris-based neural networks. Iris identification arises as an imperative subject matter in the field of biometrics and has stunning potential in real-life applications. In this paper, literature surveys over a wide range of segmentation approaches that are being used for the detection of iris are covered. The paper highlighted the use of Neural Networks for the recognition of the iris. We focus on the current research works that have been carried in the field of bioinformatics used for the identity of an individual. The process of iris identification systems like iris localization, normalization, and enhancement are covered in depth. It is very difficult to design a biometric system that identifies a person based on iris identification. However, there are some machine Learning and segmentation approaches that make the iris detection process easier.

\section{References}

[1] Jain, A.K., Bolle, R. and Pankanti, S. eds., 2006. Biometrics: personal identification in networked society (Vol. 479). Springer Science \& Business Media.

[2] Daugman, J., 2007. New methods in iris recognition. IEEE Transactions on Systems, Man, and Cybernetics, Part B (Cybernetics), 37(5), pp.1167-1175.

[3] Daugman, J., 2009. How iris recognition works. The essential guide to image processing (pp. 715739). Academic Press.

[4] Tobji, R., Di, W., and Ayoub, N., 2019. FMnet: Iris Segmentation and Recognition by Using Fully and Multi- Scale CNN for Biometric Security. Applied Sciences, 9(10), p.2042.

[5] Zhang, W. and Jia, H., Research on Convolution Neural Network in Iris Recognition Technology. In 2nd International Conference on Sensor Network and Computer Engineering (I.C.S.N.C.E. 2018).

[6] Sequeira, A.F., Murari, J. and Cardoso, J.S., 2014, January. Iris liveness detection methods in mobile applications. In 2014 International Conference on Computer Vision Theory and Applications (V.I.S.A.P.P.) (Vol. 3, pp. 22-33). IEEE. 
[7] Mozumder, A.I., and Begum, S.A., 2017. Iris Recognition Using Modular Neural Network and Fuzzy Inference System Based Score Level Fusion. International Journal of Advanced Research in Computer Science, 8(7).

[8] Hajari, K., Gawande, U. and Golhar, Y., 2016. Neural network approach to iris recognition in noisy environment. Procedia Computer Science, 78(C), pp.675-682.

[9] Kulkarni, S.B., Kulkarni, U.P. and Tushara, S., Iris Recognition using Color Models with Artificial Neural Network

[10] Chowhan, S.S. and Shinde, G.N., 2011. Iris recognition using fuzzy min-max neural network. International Journal of Computer and Electrical Engineering, 3(5), p.743.

[11] Bazrafkan, S., Thavalengal, S. and Corcoran, P., 2018. An end to end deep neural network for iris segmentation in unconstrained scenarios. Neural Networks, 106, pp.79-95.

[12] Hilal, A., Daya, B. and Beauseroy, P., 2012, October. Improved Iris Recognition using Parabolic Normalization and Multi-layer Perceptron Neural Network. In I.J.C.C.I. (pp. 643-646). [11] Gautam, Y.G.E. and Tomar, S., Iris Recognition by Neural Network. In International Conference on Advanced Computing.

[13] Peter, A., Revathi, N. and Mercy, A.P.M.M., 2013. Neural network based Matching approach for Iris recognition. International Journal of Advanced Research in Computer Engineering \& Technology (I.J.A.R.C.E.T.), 2(2), pp.618-623.

[14] Wildes, R.P., Asmuth, J.C., Green, G.L., Hsu, S.C., Kolczynski, R.J., Matey, J.R. and McBride, S.E., 1996. A machine-vision system for iris recognition. Machine vision and Applications, 9(1), pp.18.

[15] Wildes, R.P., 1997. Iris recognition: an emerging biometric technology. Proceedings of the IEEE, 85(9), pp.1348-1363.

[16] Abiyev, R.H. and Altunkaya, K., 2008. Personal iris recognition using neural network. International Journal of Security and its Applications, 2(2), pp.41-50.

[17] Rawate, K.R. and Tijare, P.A., 2017. Human Identification Using IRIS Recognition.

[18] Masek, L., 2003. Recognition of human iris patterns for biometric identification (Doctoral dissertation, Master's thesis, University of Western Australia).

[19] Meetei, T.C. and Begum, S.A., 2013, September. Performance evaluation of feature selection methods for ANN based iris recognition. In 2013 1st International Conference on Emerging Trends and Applications in Computer Science (pp. 208-213). IEEE.

[20] Daugman, J., 2007. New methods in iris recognition. IEEE Transactions on Systems, Man, and Cybernetics, Part B (Cybernetics), 37(5), pp.1167-1175.

[21] Dias, U., Frietas, V., Sandeep, P.S. and Fernandes, A., 2010. A neural network based iris recognition system for personal identification. I.C.T.A.C.T. Journal On Soft Computing, 1(2), pp.7884.

[22]Alankar, B., Yousf, N. and Ahsaan, S.U., 2020. Predictive Analytics for Weather Forecasting Using Back Propagation and Resilient Back Propagation Neural Networks. In New Paradigm in Decision Science and Management (pp. 99-115). Springer, Singapore.

[23] Sharma, S., Agrawal, S. and Silakari, S., 2014. Computational intelligence techniques used in iris recognition: a survey. International Journal of Computer Science and Information Security, 12(3), p.38. [24] AL-Allaf, O.N.A., AbdAlKader, S.A. and Tamimi, A.A., 2013. Pattern recognition neural network for improving the performance of iris recognition system. Journal of Scientific and Engineering Research, 4.

[25] Vatsa, M., Singh, R. and Noore, A., 2008. Improving iris recognition performance using segmentation, quality enhancement, match score fusion, and indexing. IEEE Transactions on Systems, Man, and Cybernetics, Part B (Cybernetics), 38(4), pp.1021-1035.

[26] Gulmire, K. and Ganorkar, S., 2012. Iris recognition using Gabor wavelet. International Journal of Engineering, 1(5).

[27] Daugman, J.G., 1993. High confidence visual recognition of persons by a test of statistical independence. IEEE transactions on pattern analysis and machine intelligence, 15(11), pp.1148-1161 